\title{
Repair of a gastrocolic fistula using a wire-guided, simultaneous dual scope approach
}
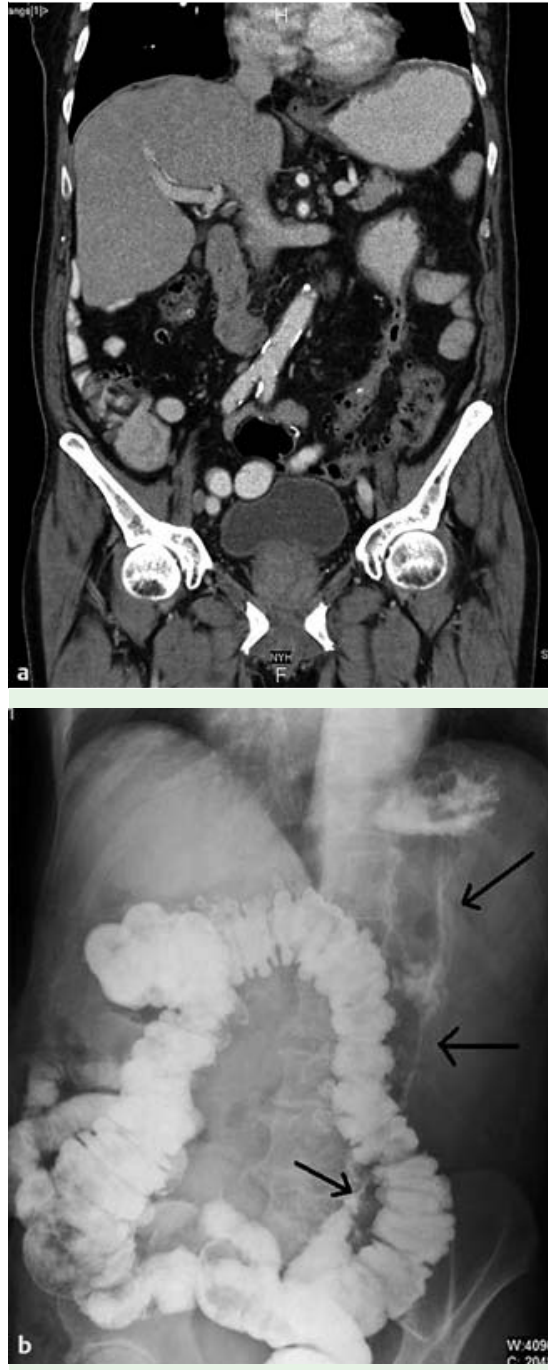

Fig. 1 Imaging findings in a 72-year-old man with a history of diverticulitis presenting with and fever and weight loss. a Barium imaging. b Computed tomography (CT) view.

A 72-year-old man with a history of diverticulitis 1 year previously, requiring a partial colectomy, presented to our hospital complaining of fever and weight loss over the preceding 3 weeks. Blood cultures revealed Gram-negative bacteremia. Antibiotics were initiated and a complete workup for infectious diseases was done without any success. Barium imaging and computed tomography (CT) of his abdomen revealed a $14-\mathrm{cm}$ long fistulous tract extending from the greater curvature of the stomach to the area of the sigmoid colon ( Fig. 1). It was felt that the

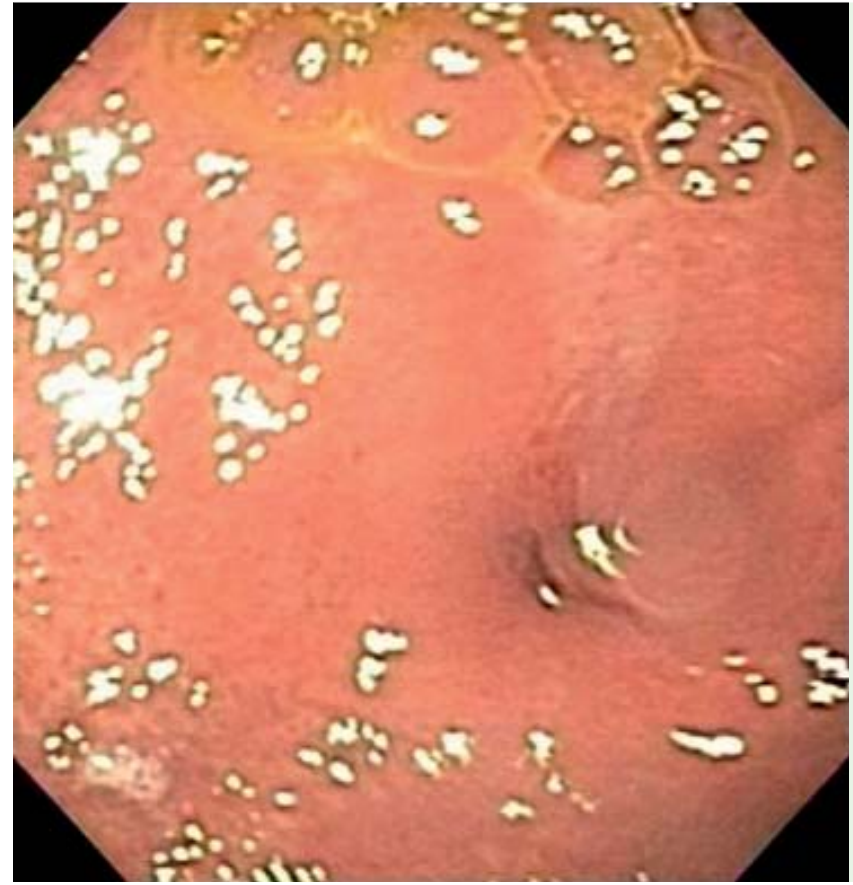

Fig. 2 Endoscopic view showing the gastric fistula orifice on the greater curvature of the stomach.

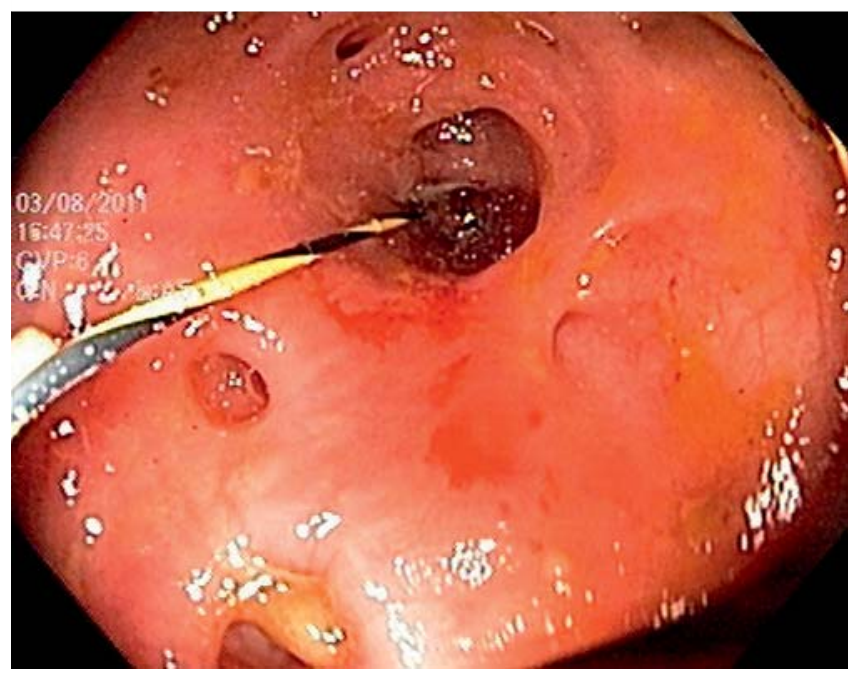

Fig. 3 A $0.025-\mathrm{cm} \mathrm{Jag}$ wire being threaded into the fistula under fluoroscopic guidance.

bacteremia was most likely due to the gastrocolic fistula and endoscopic closure was planned. At upper endoscopy, the gastric fistula opening was visualized on the greater curvature of the gastric body ( Fig.2). After injection of contrast into the tract, a 0.025-cm Jag wire (Boston Scientific, Natick, Massachusetts, USA) was threaded into the fistula under fluoroscopic guidance ( Fig.3). Colonoscopy was carried out simultaneously to visualize the guide wire's exit point, which was emerging out of a field of diverticula in the sigmoid colon. The fistula was then flushed with sterile water and a cytobrush (Cytomax II Double Lumen Cytology Brush, Wilson-Cook Medical, WinstonSalem, North Carolina, USA) was passed over the guide wire to denude the mucosa and promote closure. Lastly, both the proximal and distal ends of the fistula were closed with Resolution clips (Boston Scientific). The patient tolerated the procedure well. A repeat upper gastrointesti- 


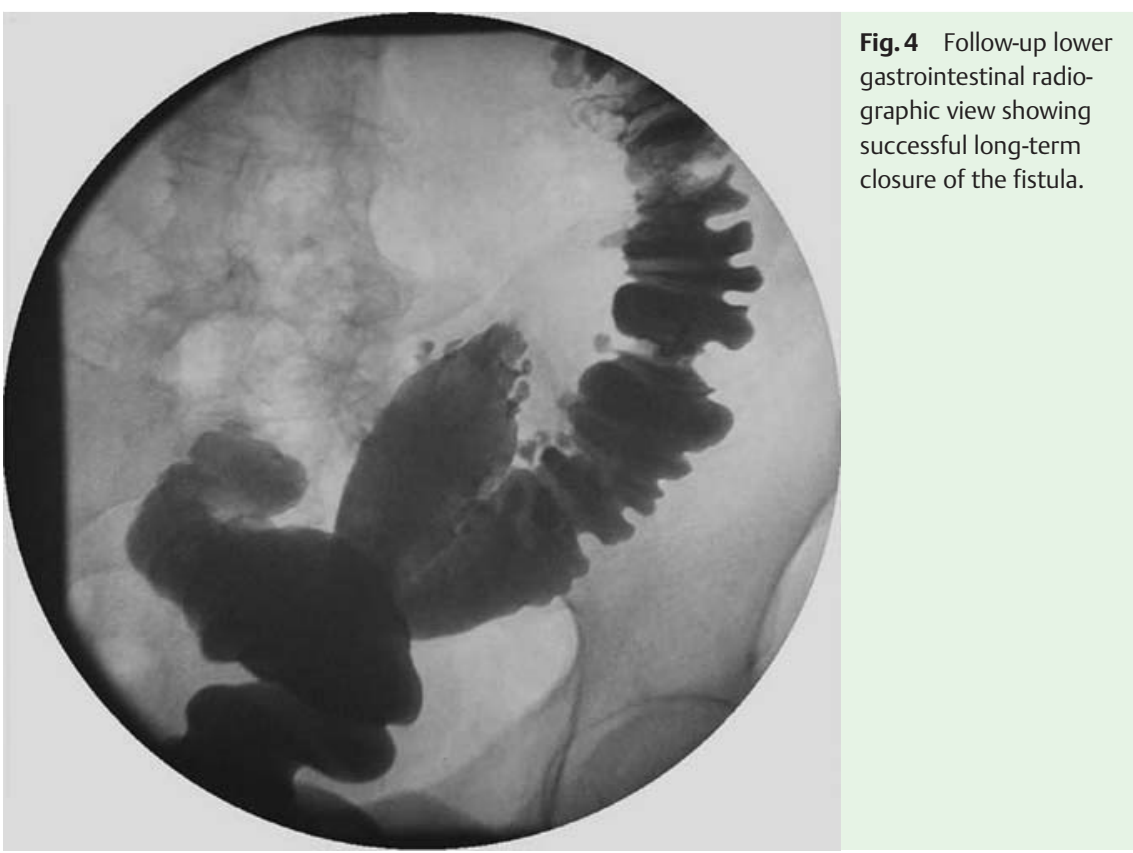

nal series performed 4 days later failed to show the fistula and the patient was discharged. At 3 months, a lower gastrointestinal series confirmed successful longterm closure ( $\bullet$ Fig. 4). To our knowledge this is the first reported case of a long gastrocolic fistula being repaired via this novel method of dual endoscopic closure with use of Resolution clips.

Endoscopy_UCTN_Code_TTT_1AQ_2AG

Competing interests: None

A. Nici, S. Hussain, M. Rubin, S. Kim

Department of Gastroenterology, New York Hospital, Flushing, New York, USA

\section{Bibliography}

DOI http://dx.doi.org/

10.1055/s-0032-1325971

Endoscopy 2013; 45: E307-E308

(c) Georg Thieme Verlag KG

Stuttgart · New York

ISSN 0013-726X

Corresponding author

\section{A. Nici, MD}

Department of Gastroenterology

New York Hospital

56-45 Main Street

Flushing

NY 11355

USA

Fax: +1-718-6707021

Anthony.Nici@gmail.com 